\title{
Echinococcus granulosus protoscolex in a patient with a pulmonary hydatid cyst
}

\author{
Protoscolex d'Echinococcus granulosus \\ chez un patient atteint de kyste hydatique pulmonaire
}

\author{
Miguel Aliste-Fernández \\ Paula San-José \\ Clinical Laboratory, Microbiology \\ Department, Parc Taulí Hospital \\ Universitari, Institut d'Investigació \\ i Innovació Parc Taulí I3PT, \\ Universitat Autònoma de Barcelona, \\ Sabadell, Spain
}

Article received October 27, 2019

accepted May 15, 2020

A 19-year-old man presented to the emergency department with a 2-month history of left costal pain exacerbated with breathing. Previously, he had worked as a shepherd and lived with a dog. Laboratory investigations revealed hyperleukocytosis (14930 leukocytes $/ \mathrm{mm}^{3}$, neutrophils $88.8 \%$ and eosinophils 0\%). Routine blood chemistry such as renal tests and liver enzymes were within normal ranges. Computed tomography of the chest revealed a large unilocular cyst of $8 \times 8 \mathrm{~cm}$ in size in the left lung's lower lobe (figure 1A). Surgical removal of cyst allowed sampling of the cystic content. Microscopic examination confirmed the presence of many protoscolices that were diagnostic of the tapeworm Echinococcus granulosus (figure 1B). Human cystic echinococcosis (CE, also called hydatidosis, or hydatid disease) is caused by the larval stages of Echinococcus granulosus sensu lato [1]. Humans become infected by ingesting eggs released by the definitive host (dog), leading to the development of cysts in various organs. It is present worldwide, being more frequently in rural and grazing areas. Cystic echinococcosis remains silent for years before the enlarging cysts cause symptoms in the affected organs. Immunodiagnostic tests can be very helpful in the diagnosis particularly in conjunction with imaging, and should be used before invasive methods. Unfortunately, serological methods for testing CE patients have 60 to $90 \%$ sensitivity, increasing the false negative rate up to $40 \%$ [1]. Various options for patient's management are used, depending on cyst stage. It includes, alone or combined, either surgery, non-surgical procedures, albendazole therapy or "watch and wait" approach. Our patient was treated with albendazole for 1 month with no complications at follow-up. 


\section{Biological pictures}

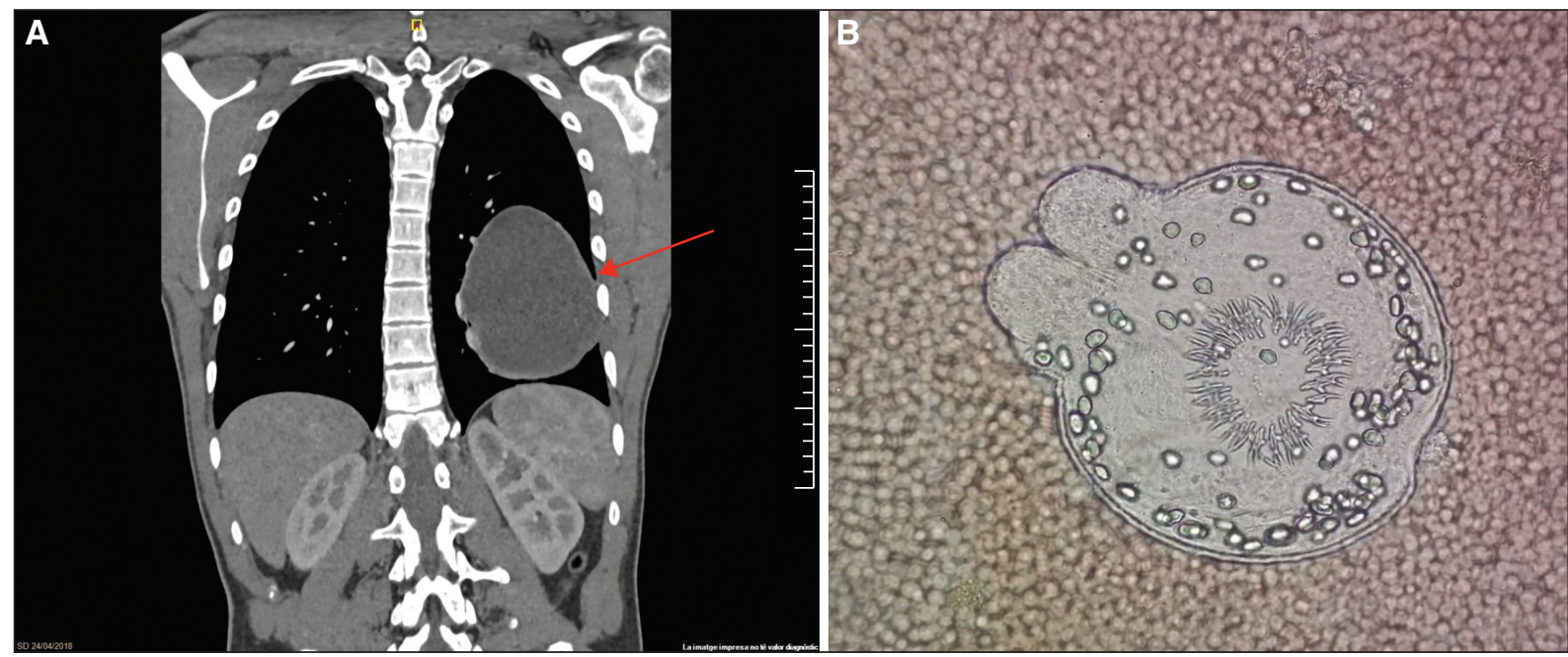

Figure 1. (A) Computed tomography of the chest with the hydatid cyst in the left lung (arrow). (B) Echinococcus granulosus protoscolex in the cystic content observed under the microscope at 400x magnification (surrounded by many red blood cells).

Conflict of interest: none of the authors has any conflict of interest to disclose concerning this article.

\section{Reference}

1. Wen H, Vuitton L, Tuxun T, Li J, Vuitton DA, Zhang W, et al. Echinococcosis: advances in the 21st Century. Clin Microbiol Rev 2019 ; 32(2) : e00075-18. 